\title{
VARIATIONAL INEQUALITIES OF STRONGLY NONLINEAR ELLIPTIC OPERATORS OF INFINITE ORDER
}

\section{ADELL T. EL-DESSOUKY}

Department of Mathematics

Faculty of Science

Helwan University

Cairo, Egypt

(Received June 30, 1992 and in revised form December 31, 1992)

\begin{abstract}
The present paper deals with the study of the solvability of variational inequalities for strongly nonlinear elliptic operators of infinite order with liberal growth on the coefficients.

KEY WORDS AND PHRASES: Variational inequalities of strongly nonlinear problems, Sobolev and Sobolev Orlicz spaces of infinite order.
\end{abstract}

1992 AMS SUBJECT CLASSIFICATION CODES: 35G30, 46E35.

\section{INTRODUCTION}

In a series of articles Dubinskii [1,2] considered the nontriviality of Sobolev spaces of infinite order corresponding to boundary value problems for linear differential equations of infinite order and obtained the solvability of these problems for the case in which the coefficients of the equation grow polynomially with respect to the derivatives.

Chan [3], extended the results of Dubinskii to include the case of operators with rapidly (slowly) increasing coefficients.

In this paper we generalize the above results to cover the solvability of variational inequalities for strongly nonlinear operators of the form

$$
A u(x)+B u(x), \quad x \in \Omega
$$

where $\Omega$ is a bounded domain in $R^{n}$ and

$$
\begin{aligned}
& A u(x)=\sum_{|\alpha|=0}^{\infty}(-)^{|\alpha|} D^{\alpha} A_{\mathrm{\alpha}}\left(x, D^{\gamma} u(x)\right), \quad|\gamma| \leq|\alpha|, \\
& B u(x)=\sum_{|\alpha|=M}(-)^{|\alpha|} D^{\alpha} B_{\mathrm{\alpha}}\left(x, D^{\alpha} u(x)\right), \quad M \text { fixed, }
\end{aligned}
$$

with more liberal growth on the coefficients.

\section{PRELIMINARIES}

Let $\Omega$ be a bounded domain in $\mathbf{R}^{n}(n \geq 2)$ for which the cone and the strong local Lipschitz properties hold [4].

An $N$-function is any continuous map $\Phi: \mathbf{R} \rightarrow \mathbf{R}$ which is even, convex and satisfies $\Phi(t) / t \rightarrow 0$ (resp. $+\infty$ ) as $t \rightarrow 0$ (resp., $+\infty$ ). The conjugate or complementary $N$-function of $\Phi$ and its nonnegative reciprocal will be denoted by $\bar{\Phi}$ and $\Phi^{-1}$, respectively [4].

When $\Phi$ and $\Psi$ are two $N$-functions, we shall write $\Psi<\Phi$ if for any $\varepsilon>0$

$$
\operatorname{Lim}_{t \rightarrow \infty} \Psi(t) / \Phi(\varepsilon t)=0
$$

The Orlicz space $L_{\Phi_{\alpha}}(\Omega)$ corresponding to $N$-functions $\Phi_{\alpha}$ is defined as the set of all measurable functions $u: \Omega \rightarrow \mathbf{R}$ such that 


$$
\|u\|_{\Phi_{\alpha}}=\inf \left\{\lambda>0 ; \int_{\Omega} \Phi_{\alpha}(u / \lambda) \leq 1\right\}<\infty
$$

Let $E_{\Phi_{\alpha}}(\Omega)$ be the (norm) closure in $L_{\Phi_{\alpha}}(\Omega)$ of $L^{\infty}(\Omega)$-functions with compact support in $\bar{\Omega}$.

The Sobolev-Orlicz spaces of functions $u$ such that $u$ and its distributional derivatives $D^{\alpha} u,|\alpha| \leq m$, lie in $L_{\Phi_{\alpha}}(\Omega)\left(\right.$ resp. $\left.E_{\Phi_{\alpha}}(\Omega)\right)$. These are Banach spaces with the norm

$$
\|u\|_{m, \Phi_{\alpha}}=\left(\sum_{|\alpha| \leq m}\left\|D^{\alpha} u\right\|_{\Phi_{\alpha}}^{2}\right)^{1 / 2}
$$

and they are identified to subspaces of the product

$$
\prod_{|\alpha| \leq m} L_{\Phi_{\alpha}}(\Omega)-\Pi L_{\Phi_{\alpha}}
$$

Denote by $C^{\infty}(\Omega)$ the space of infinitely differentiable functions on $\Omega, D(\Omega)$ the space $C^{\infty}(\Omega)$ with compact support in $\Omega$ and by $\mathcal{D}^{\prime}(\Omega)$ for the space of distributions on $\Omega$.

We define $W_{0}^{m} L_{\Phi_{a}}(\Omega)$ as the o $\left(\Pi L_{\Phi_{a}}, \Pi E_{\Phi_{a}}\right)$ closure of $D(\Omega)$ in $W^{m} L_{\Phi_{a}}(\Omega)$ and $W_{0}^{m} E_{\Phi_{a}}(\Omega)$ as the norm closure of $D(\Omega)$ in $W^{m} L_{\Phi_{a}}(\Omega)$.

The Sobolev-Orlicz spaces of infinite order is defined by:

$$
W^{\infty} L_{\phi_{a}}(\Omega)=\left\{u \in C^{\infty}(\Omega): \sum_{|\alpha|-0}^{\infty} \int_{\Omega} \Phi_{\alpha}\left(D^{\alpha} u(n)\right) d x<\infty\right\},
$$

and $x \in \Omega$. Moreover there exists a function $h_{1} \in L^{1}(\Omega)$, independent of $l$, and a sequence of positive numbers $\left(S_{l}\right)_{l \in N}$ with $\sum_{l} \lambda_{1} S_{l}<\infty$ such that

$$
\sup _{\left|\xi_{\gamma}\right| \leq S_{l}^{-1}}\left|A_{\alpha}\left(x, \xi_{Y}\right)\right| \leq h_{1}(x) S_{l} .
$$

(A1) There exists a constant $C_{0}>0$ and a function $h_{2} \in L^{1}(\Omega)$, both independent of $l$, such that

$$
\sum_{|\alpha|=0}^{l} A_{\alpha}\left(x, \xi_{\gamma}\right) \xi_{\alpha} \geq C_{0} \sum_{|\alpha|=0}^{l} a_{\alpha}\left|\xi_{\alpha}\right|^{P}-h_{2}(x)
$$

for all $x \in \Omega, \xi_{\gamma} \in \mathbf{R}^{\lambda_{1}}$.

(A2) For all $l \in N$, a.a. $x \in \Omega$ and all distinct $\xi_{\gamma}, \xi_{\gamma}^{*} \in \mathbf{R}^{\lambda_{1}}$

$$
\sum_{|\alpha|=0}^{l}\left(A_{\alpha}\left(x, \xi_{\gamma}\right)-A_{\alpha}\left(x, \xi_{\gamma}^{*}\right)\right)\left(\xi_{\alpha}-\xi_{\alpha}^{*}\right)>0 \text {. }
$$

Or the following one:

(A1)* For all $l \in \mathrm{N}$, each $A_{\alpha}\left(x, \xi_{\gamma}\right)$ is a real-valued Caratheodory function defined on $\Omega \times \mathbf{R}^{\lambda_{1}}$. There exist two $N$-functions $\Phi_{\alpha}, \Psi_{\alpha}$ with $\Psi_{\alpha} \ll \Phi_{\alpha}$; functions $a_{\alpha}(x)$ in $E_{\bar{\Phi}_{\alpha}}(\Omega)$ for $|\alpha|=l$, in $L_{\bar{\Phi}_{\alpha}}(\Omega)$ for $|\alpha|<l$; and positive constants $c_{1}, c_{2}$, both independent of $l$, such that if $|\alpha|=l$

$$
\left|A_{\alpha}\left(x, \xi_{\gamma}\right)\right| \leq a_{\alpha}(x)+c_{1} \sum_{|\beta|-l} \bar{\Phi}_{\alpha}^{-1} \Phi_{\alpha}\left(c_{2} \xi_{\beta}\right)+c_{1} \sum_{|\beta|<l} \bar{\Psi}_{\alpha}^{-1} \Phi_{\alpha}\left(c_{2} \xi_{\beta}\right),
$$

if $|\alpha|<l$

$$
\left|A_{\alpha}\left(x, \xi_{\gamma}\right)\right| \leq a_{\alpha}(x)+c_{1} \sum_{|\beta|<l} \bar{\Phi}_{\alpha}^{-1} \Psi_{\alpha}\left(c_{2} \xi_{\beta}\right)+c_{1} \sum_{|\beta|<l} \bar{\Phi}_{\alpha}^{-1} \Phi_{\alpha}\left(c_{2} \xi_{\beta}\right),
$$

for a.a. $x \in \Omega$ and all $\xi_{\gamma} \in \mathbf{R}^{\lambda_{1}}$. 
(A2)* There exist functions $b_{\alpha}$ in $E_{\bar{\Phi}_{\alpha}}(\Omega)$ for $|\alpha|=l$, in $L_{\bar{\Phi}_{a}}(\Omega)$ for $|\alpha|<l$; function $h_{3} \in L^{1}(\Omega)$ and positive constants $d_{1}, d_{2}$, independent of $l$, such that

$$
\sum_{|\alpha|-0}^{l} A_{\alpha}\left(x, \xi_{\gamma}\right) \xi_{\alpha} \geq d_{1} \sum_{|\alpha|=0}^{l} \Phi_{\alpha}\left(d_{2} \xi_{\alpha}\right)-\sum_{|\alpha|=0}^{l} b_{\alpha}(x) \xi_{\alpha}-h_{3}(x)
$$

for a.a. $x \in \Omega$ and all $\xi_{y} \in \mathbf{R}^{\lambda_{1}}$

$$
W_{0}^{\infty} L_{\Phi_{a}}(\Omega)=\left\{u \in D(\Omega):\|u\|_{\infty, \Phi_{a}}=\sum_{|a|-0}^{\infty}\left\|D^{a} u\right\|_{\Phi_{a}}<\infty\right\}
$$

They are Banach spaces with the norm $\|\cdot\|_{\infty, \Phi_{a}}$.

Similar definition of $W_{0}^{\infty} E_{\Phi_{a}}(\Omega)$ is obvious. The dual of $W_{0}^{\infty} L_{\Phi_{\alpha}}(\Omega)\left(\right.$ resp. $W_{0}^{\infty} E_{\Phi_{a}}(\Omega)$ ) will be denoted by $W^{-\infty} E_{\bar{\Phi}_{a}}(\Omega)\left(\right.$ resp. $\left.W^{-\infty} L_{\bar{\Phi}_{a}}(\Omega)\right)$, where

$$
\left.W^{-\infty} E_{\bar{\Phi}_{\alpha}}(\Omega)\right)\left(\operatorname{resp} . W^{-\infty} L_{\bar{\Phi}_{\alpha}}(\Omega)\right)=\left\{h \in \mathcal{D}^{\prime}(\Omega): h(x)=\sum_{|\alpha|=0}^{\infty}(-)^{|\alpha|} D^{\alpha} h_{\alpha}, h_{\alpha} \in E_{\bar{\Phi}_{\alpha}}(\Omega)\left(\operatorname{resp} . L_{\Phi_{\alpha}}(\Omega)\right)\right\}
$$

These spaces are Banach spaces with the norm

$$
\|h\|_{-\infty, \bar{\Phi}_{a}}=\sum_{|\alpha|=0}^{\infty}\left\|h_{\alpha}\right\|_{\bar{\Phi}_{a}}<\infty
$$

The duality of $W_{0}^{\infty} L_{\Phi_{\alpha}}(\Omega)$ and $W^{-\infty} E_{\bar{\Phi}_{\alpha}}(\Omega)$ is defined by

$$
\langle h, u\rangle=\sum_{|\alpha|-0}^{\infty} \int_{\Omega} h_{\alpha}(x) D^{\alpha} u(x) d x .
$$

Let $1 \leq p<\infty$. The Sobolev spaces of infinite order are defined by

$$
W_{0}^{\infty}\left(a_{\alpha}, p\right)(\Omega)=\left\{u \in \mathcal{D}(\Omega):\|u\|_{\infty, p}^{p}=\sum_{|\alpha|=0}^{\infty} a_{\alpha} \int_{\Omega}\left|D^{\alpha} u(x)\right|^{p} d x<\infty\right\},
$$

where $a_{\alpha} \geq 0$ is a sequence of numbers. We formally define the spaces dual to $W_{0}^{\infty}\left(a_{\alpha}, p\right)(\Omega)$ via:

$$
W^{-\infty}\left(a_{\alpha}, p^{\prime}\right)(\Omega)=\left\{h: h=\sum_{|\alpha|=0}^{\infty}(-)^{|\alpha|} a_{a} D^{\alpha} h_{\mathrm{a}} ; h_{\mathrm{a}} \in L^{p^{\prime}}(\Omega):\|h\|_{-\infty, p^{\prime}}^{p^{\prime}}=\sum_{|\alpha|=0}^{\infty} a_{\mathrm{a}}\left\|h_{\mathrm{a}}\right\|_{L^{p^{\prime}(\Omega)}}^{p^{\prime}}<\infty\right\}, p^{\prime}=p \rightarrow p / p-1
$$

For more details we may refer to $[1,3,4]$. Let $l, M \in N, M$ being fixed. By $\lambda_{1}$ and $\lambda_{2}$ we denote the number of multi-indices $\alpha$ with $|\alpha| \leq l,|\alpha| \leq M$, respectively.

\section{CONDITIONS ON THE COEFFICIENTS}

To define the operator (1.2) more precisely we introduce either the following set of hypotheses:

(A3) For all $l \in N$ and $|\gamma| \leq|\alpha|$, each $A_{\alpha}\left(x, \xi_{\gamma}\right)$ is a Caratheodory function, i.e., $A_{\alpha}\left(x, \xi_{\gamma}\right)$ is measurable in $x \in \Omega$ for all fixed $\xi_{\gamma} \in \mathbf{R}^{\lambda_{1}}$, and continuous in $\xi_{\gamma}$ for almost all (a.a.) fixed.

(A2)* As in (A2). For the operator (1.3) we impose the following assumption:

(B1) $B_{\alpha}\left(x, \xi_{\alpha}\right)$ is a Caratheodory function defined on $\Omega \times \mathbf{R}^{\lambda_{2}}$. There exists a function $h_{4}$ in $L^{1}(\Omega)$ such that:

$$
\left|B_{\alpha}\left(x, \xi_{\alpha}\right)\right| \leq h_{4}(x) P_{\alpha}\left(\xi_{\alpha}\right)
$$

for some continuous function $P_{\alpha}: \mathbf{R}^{\lambda_{2}} \rightarrow \mathbf{R}$ and

$$
B_{\alpha}\left(x, \xi_{\alpha}\right) \xi_{\alpha} \geq 0, \quad x \in \Omega,|\alpha| \leq M
$$




\section{MAIN RESULTS}

THEOREM 4.1. Let $K$ be a closed convex subset of $W_{0}^{\infty}\left(a_{\alpha}, p\right)(\Omega)$ containing the origin. Suppose that (A2)-(A3) and (B1) hold. Let $f \in W^{-\infty}\left(a_{\alpha}, p^{\prime}\right)(\Omega)$ be given. Then there exists at least one solution $u \in K$ of

$$
\langle A(u), v-u\rangle+\langle B(u), v-u\rangle \geq\langle f, v-u\rangle \quad \forall v \in K
$$

PROOF. Consider a partial sum of order $2 l$ of the series (4.1):

$$
\left\langle A_{2 l}\left(u_{l}\right), v-u_{l}\right\rangle+\left\langle B\left(u_{l}\right), v-u_{l}\right\rangle \geq\left\langle f^{l}, v-u_{l}\right\rangle \quad \forall v \in K
$$

where

$$
\begin{aligned}
A_{2 l}\left(u_{l}\right)(x) & =\sum_{|\alpha|=0}^{l}(-)^{|\alpha|} D^{\alpha} A_{\alpha}\left(x, D^{\gamma} u_{l}\right), \quad|\gamma| \leq|\alpha|, \\
B\left(u_{l}\right)(x) & =\sum_{|\alpha|} \sum_{s=l}(-)^{|\alpha|} D^{\alpha} B_{\alpha}\left(x, D^{\alpha} u_{l}\right),
\end{aligned}
$$

and

$$
f^{l}=\sum_{|\alpha|=0}^{l}(-)^{|\alpha|} a_{\alpha} D^{\alpha} f_{\alpha} \in W^{-1}\left(a_{\alpha}, p^{\prime}\right)(\Omega) .
$$

For the solvability of (4.2), in view of (A2)-(A3) and (B1), we refer to [5] and [6].

Put $v=0$ in (4.2), and use (A2) and (B1) to get the a priori-bound

$$
\left\|u_{l}\right\|_{W_{0}^{\prime}\left(a_{a}, p\right)(\Omega)} \leq \text { const. }
$$

Since $u_{l} \in W^{\prime}\left(a_{\alpha}, p\right)(\Omega)$ implies $u_{l} \in W^{1}\left(a_{\alpha}, p\right)(\Omega)$ we get from the compactness of

$$
W^{1}\left(a_{\alpha}, p\right)(\Omega) \rightarrow C(\bar{\Omega}),
$$

the uniform convergence of $u_{l}(x) \rightarrow u(x)$ on $\bar{\Omega}$ as $l \rightarrow \infty$. Similarly, by the compactness of

$$
W^{l}\left(a_{\alpha}, p\right)(\Omega) \rightarrow C^{l-m}(\bar{\Omega}), \quad \text { for large enough } l \text { and } m \in \mathbf{N} ;
$$

we obtain,

$$
D^{\alpha} u_{l}(x) \rightarrow D^{\alpha} u(x) \quad \text { uniformly on } \bar{\Omega} \text { as } l \rightarrow \infty
$$

Using the definition of $W_{0}^{\infty}\left(a_{\alpha}, p\right)(\Omega)$ we get $u \in W_{0}^{\infty}\left(a_{\alpha}, p\right)(\Omega)$ and by the closedness of $K, u \in K$.

It remains to show that $u$ is a solution of (4.1). For this purpose it suffices to prove the assertions:

$$
\begin{gathered}
\operatorname{Lim}_{l}\left\langle A_{2 l}\left(u_{l}\right), z\right\rangle=\langle A(u), z\rangle \\
\operatorname{Lim}_{l}\left\langle B\left(u_{l}\right), z\right\rangle=\langle B(u), z\rangle \\
\operatorname{Liminf}_{l}\left\langle A_{2 l}\left(u_{l}\right), u_{l}\right\rangle \geq\langle A(u), u\rangle
\end{gathered}
$$

and

$$
\underset{l}{\operatorname{Liminf}}\left\langle B\left(u_{l}\right), u_{l}\right\rangle \cdot \geq\langle B(u), u\rangle
$$

for all $z \in K$.

To show (4.4) we use the inequality:

$$
\left|A_{\alpha}\left(x, D^{\gamma} u_{l}\right)\right| \leq \sup _{\left|\xi_{\gamma}\right|<S_{l}^{-1}}\left|A_{\alpha}\left(x, \xi_{\gamma}\right)\right|+S, A_{\alpha}\left(x, D^{\gamma} u_{l}\right) D^{\alpha} u_{l}
$$


as well as the uniform boundedness of $\left\{\left\langle A_{2 l} u_{l}, u_{l}\right\rangle\right\}$ in $L^{1}(\Omega)$, to obtain the uniform equi-integrability of $\left\{A_{\mathrm{a}}\left(x, D^{\mathrm{y}} u_{l}\right)\right\}$ in $L^{1}(\Omega)$ provided that $\sum S_{l} \lambda_{1}(\cdot, l)<\infty$. Now, in view of Vitali's convergence theorem, (4.7) follows.

To show (4.5) we have

$$
\sum_{|\alpha| \leq M} \int_{\Omega}\left|B_{\alpha}\left(x, D^{\alpha} u_{l}\right)\right| \leq \int_{\Omega}\left|h_{4}(x) P_{\alpha}\left(D^{\alpha} u_{l}\right)\right| \leq\left\|h_{4}\right\|_{L^{1}(\Omega)} \sum_{|\alpha| \leq M}\left\|P_{\alpha}\left(D^{\alpha} u_{l}\right)\right\|_{L^{-(}(\Omega)} \leq \text { const. }
$$

and (4.5) follows from the dominated convergence theorem. Assertions (4.6) and (4.7) are direct consequences of Fatou's lemma in view of the uniform convergence (4.3) and the proof is completed.

The above result enables us to give the following theorem.

THEOREM 4.2. Let $K$ be a convex $\sigma\left(W^{\infty} L_{\Phi_{\alpha}}(\Omega), W^{-\infty} E_{\bar{\Phi}_{\alpha}}(\Omega)\right)$ sequentially closed subset of $W^{\infty} L_{\Phi_{a}}(\Omega)$ such that $K \cap W_{0}^{\infty} E_{\Phi_{a}}(\Omega)$ is $\sigma\left(W^{\infty} L_{\Phi_{a}}(\Omega), W^{-\infty} L_{\Phi_{a}}(\Omega)\right)$ dense in $K$ and $0 \in K$. Let $f \in W^{-\infty} E_{\bar{\Phi}_{a}}(\Omega)$ be given. Let the hypotheses $(\mathrm{A} 1)^{*}-(\mathrm{A} 3)^{*}$ hold. Then there exists at least one solution $u \in K$ such that:

$$
\langle A u, v-u\rangle-\langle f, v-u\rangle \geq 0 \quad \forall v \in K
$$

OUTLINE OF PROOF. As in Theorem 4.1, we may consider the auxiliary variational inequality

$$
\left\langle A_{2 m}\left(u_{m}\right), v-u_{m}\right\rangle-\left\langle f^{m}, v-u_{m}\right\rangle \geq 0 \quad \forall v \in K
$$

The solvability of (4.9) is a consequence of [7]. Thus, there exists $u_{m} \in K$ solving (4.9).

Put $v=0$ in (4.9) and make use of (A2)*, we have

$$
\int_{\Omega} \Phi_{\alpha}\left(c_{2} D^{\alpha} u_{m}\right) \leq c_{3}
$$

where

$$
c_{3}=c_{3}\left(\|f\|_{W-L_{\sigma_{a}}(\Omega)}\right)
$$

Hence, there exists a subsequence of $u_{m}$ such that $u_{m} \rightarrow u$ in $C^{\infty}(\Omega)$. By the definition of $W^{\infty} L_{\Phi_{a}}(\Omega)$ and the $\sigma\left(W^{\infty} L_{\Phi_{a}}(\Omega), W^{-\infty} E_{\bar{\Phi}}(\Omega)\right)$ sequential closedness of $K$, we get $u \in K$. To show that $u$ solving (4.8) it remains to prove assertions (4.4) and (4.6) of Theorem 4.1.

A similar procedure of Theorem 4.1 may be carried and the proof follows.

EXAMPLE. As a particular example which can be handled by Theorem 4.1 and falls outside the scope of [1], consider the nonlinear Dirichlet boundary-value problem

$$
\sum_{l=0}^{\infty} \sum_{|\alpha|-l}(-)^{|\alpha|} D^{\alpha}\left(a_{\alpha} S_{l}^{P}\left|D^{\alpha} u\right|^{P-2} D^{\alpha} u\right)+|u| e^{|u|}=f(x)
$$

where $\left(S_{l}\right)_{l \in \mathrm{N}}$ is a sequence described in (A1). In fact:

$$
\begin{gathered}
A_{\alpha}\left(x, D^{\gamma} u\right):=a_{\alpha} S_{l}^{p}\left|D^{\alpha} u\right|^{P-2} D^{\alpha} u, \quad|\gamma|=|\alpha| \\
B_{\alpha}\left(x, D^{\alpha} u\right):=|u| e^{|\psi|}
\end{gathered}
$$

By the Sobolev's embedding theorem, for $u \in W^{l}\left(a_{\alpha}, p\right)(\Omega)(l p>n)$, the functions $D^{\alpha} u$ are bounded for all $|\alpha| \leq l$. Therefore $A_{\alpha}\left(x, \xi_{\gamma}\right)$ and $B_{\alpha}\left(x, \xi_{\gamma}\right)$ are $L^{\infty}(\Omega)$-functions and hence (A1) and (B1) follow. Condition (A2) is obvious, while (A3) follows in view of the inequality

$$
|x|^{P}+|y|^{P}-x y\left(|x|^{P-2}+|y|^{P-2}\right)>0 \text { for } x \neq y
$$


Thus the hypotheses of Theorem 4.1 are satisfied. Our example falls outside the scope of [1], for the term $|u| e^{|u|}$ does not verify the polynomial growth condition of [1].

ACKNOWLEDGMENTS. The author would like to express his sincere thanks to the referee for his suggestions and comments. He also expresses his sincere thanks to the Editor Professor Lokenath Debnath for his cooperation.

\section{REFERENCES}

[1] DUBINSKII, JU. A., Sobolev spaces of infinite order and the behavior of solutions of some boundary-value problems with unbounded increase of the order of the equation, Math. USSR Sbornik, 72 (1972), 143-162.

[2] DUBINSKII, JU. A., On traces of functions in Sobolev spaces of infinite order and non-homogeneous boundary value problems, Math. USSR Sbornik, 34 (1978), 627-644.

[3] CHAN DYK VAN, Traces of functions from Sobolev-Orlicz classes of infinite order and nonhomogeneous boundary value problems for equations with arbitrary nonlinearity, Soviet Math. Dokl., 22 (1980), 626-630.

[4] ADAMS, R. A., Sobolev spaces, Academic Press, New York (1975).

[5] EL-DESSOUKY, A. T., Optimal control of systems governed by nonlinear elliptic and parabolic problems of arbitrary order, $D$. Phil. Thesis, Al-Azhar University, Cairo, Egypt (1989).

[6] FUCIK, A. and KUFNER, A., Nonlinear differential equations, Elsevier Scientific Publishing Company, Amsterdam (1980).

[7] GOSSEZ, J. P. and MUSTONEN, V., Variational inequalities in Orlicz-Sobolev spaces, Nonlinear Analysis, Theory, Methods and Applications, 11 (1987), 379-392. 


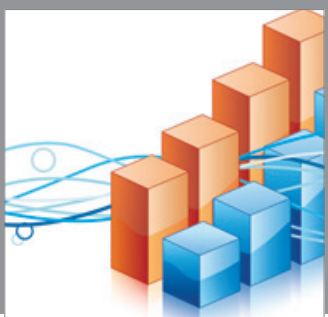

Advances in

Operations Research

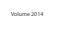

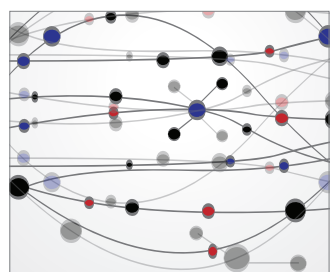

\section{The Scientific} World Journal
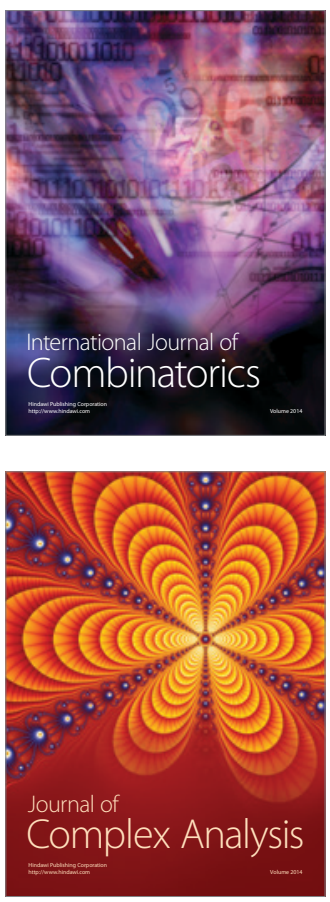

International Journal of

Mathematics and

Mathematical

Sciences
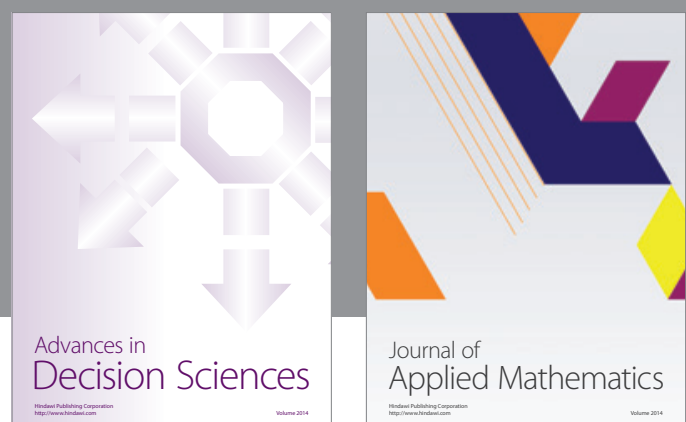

Journal of

Applied Mathematics
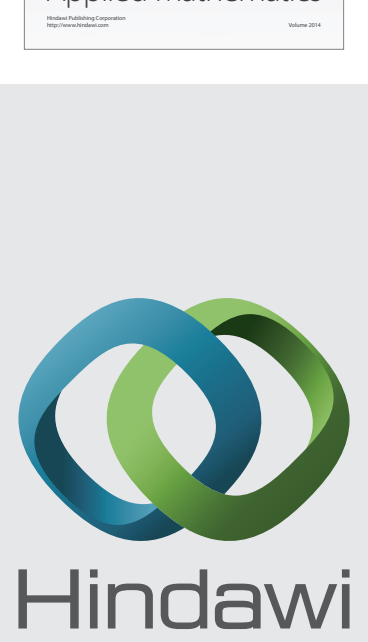

Submit your manuscripts at http://www.hindawi.com
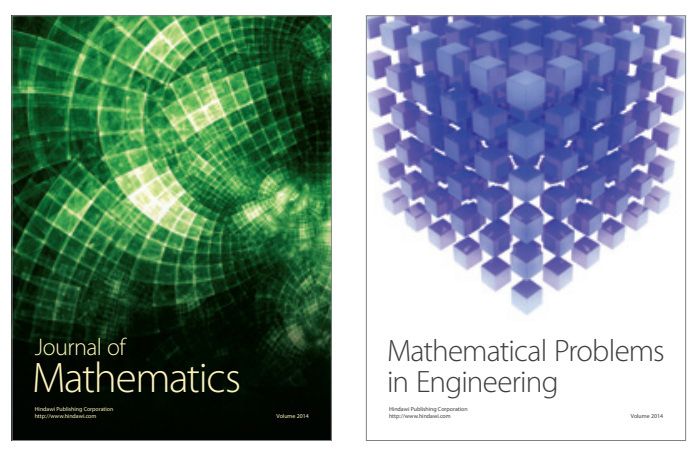

Mathematical Problems in Engineering
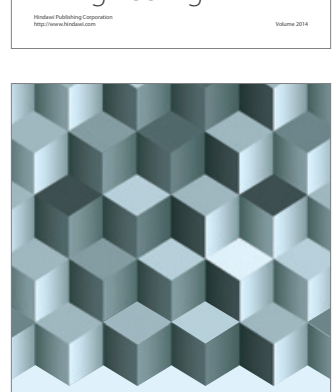

Journal of

Function Spaces
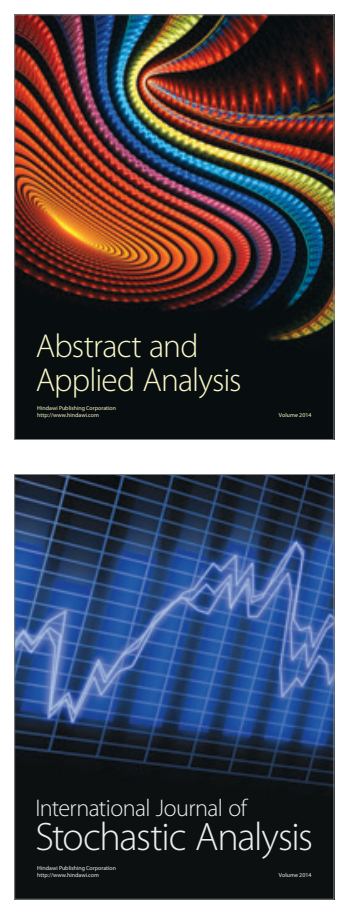

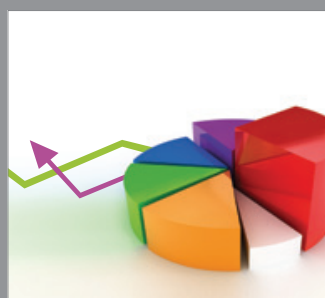

ournal of

Probability and Statistics

Promensencen
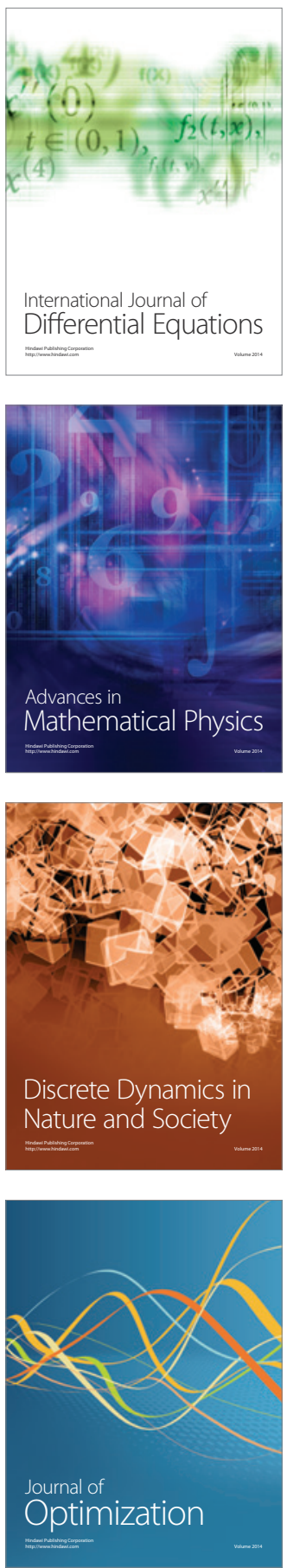